\title{
On the distribution of the nontrivial zeros of quadratic $L$-functions close to the real axis
}

by

\author{
A. E. ÖZLÜK and C. SNyder (Orono, ME)
}

1. Introduction. The object of this paper is to investigate the distribution of the nontrivial zeros, near the real axis, of quadratic $L$-functions.

Interest in the zeros of Dirichlet $L$-functions close to the real axis is well documented. For example, see [1]-[3], [7], [9]-[11]. For instance, if $\chi$ is an odd quadratic character, then the zeros of $L(s, \chi)$ close to $s=1 / 2$ influence the size of the class number of the complex quadratic number field associated with $\chi$. Also, if $\chi$ is the nonprincipal character modulo 4 , then the location of the "first" zero of $L(s, \chi)$ in the critical strip has a bearing on how the primes are distributed in the residue classes 1 and $3 \bmod 4$, respectively, and in particular, on a phenomenon first observed by Chebyshev [4] concerning the discrepancies in the distribution of primes in different residue classes. Roughly speaking, there are "more" primes congruent to $3 \bmod 4$ than congruent to $1 \bmod 4$.

In this paper, let $K(s)$ be a complex function such that $|K(1 / 2+i t)|$ is a rapidly decreasing function of $t>0$. Moreover, let $a(x)$ be its Mellin transform as defined in the next section. Define

$$
F_{K}(\alpha, D)=\left(\frac{1}{2} K\left(\frac{1}{2}\right) D\right)^{-1} \sum_{d \neq 0} e^{-\pi d^{2} / D^{2}} \sum_{\varrho(d)} K(\varrho) D^{i \alpha \gamma},
$$

where $\varrho$ ranges over the nontrivial zeros of $L\left(s, \chi_{d}\right)$, the Dirichlet $L$-function associated with the Kronecker symbol $\chi_{d}=(d / \cdot)$. Under the Generalized Riemann Hypothesis, we show that

$$
\begin{aligned}
& F_{K}(\alpha, D) \\
& \quad= \begin{cases}-1+\left(\frac{1}{2} K\left(\frac{1}{2}\right)\right)^{-1} D^{-\alpha / 2} a\left(D^{-\alpha}\right) \log D+o(1) & \text { if }|\alpha|<1, \\
0+o(1) & \text { if } 1<|\alpha|<2\end{cases}
\end{aligned}
$$

as $D \rightarrow \infty$.

1991 Mathematics Subject Classification: Primary 11M26. 
Notice that this so-called "form factor" correlates the distance of the nontrivial zeros to the real axis rather than correlating the distance between pairs of such zeros, as first investigated by H. L. Montgomery [6] for the zeros of the Riemann zeta function.

This result is a major improvement over that in [9] where we obtained information for $|\alpha|<2 / 3$ only. Our new result shows a discontinuity in $F_{K}$ at $\alpha=1$. We shall see that this discontinuity is related to the phenomenon of Chebyshev mentioned above but in a more general setting.

We then give two corollaries to the main result. One of the corollaries implies that for quadratic $L$-functions, the nontrivial zeros near the real axis are "sparser" than the expected number of such zeros. This is in direct contrast to the zero-distribution when all Dirichlet $L$-functions are taken into account. The other corollary implies that not more than $6.25 \%$ of all integers $d$ have the property that $1 / 2$ is a zero of $L\left(s, \chi_{d}\right)$. As far as we know, no quadratic $L$-function has been found with a zero at $1 / 2$. If any exist, then more than likely the density of such $d$ would be 0 .

2. Preliminaries. In this section we prove a number of lemmas which will be needed in the proof of our main theorem. We assume that $K(s)$ is an analytic function in the strip $-1<\Re s<2$ such that

$$
a(x)=\int_{c-\infty i}^{c+\infty i} K(s) x^{-s} d s
$$

is absolutely convergent for $-1<c<2$ and for all $x>0, K(1 / 2+i t)=$ $K(1 / 2-i t)$, and $a(x)$ is continuously differentiable, of bounded variation, real-valued, and of compact support on the interval $(0, \infty)$. More specifically, we assume $a(x)=0$ outside $[A, B]$ for some $0<A<B<\infty$. Finally, we recall the identity

$$
K(s)=\int_{0}^{\infty} a(t) t^{s} \frac{d t}{t}
$$

Lemma 1. $\sum_{d} e^{-\pi d^{2} / y^{2}}=y+o(1)$ as $y \rightarrow \infty$. (Here $\sum_{d}$ denotes the sum over all nonzero integers $d$.)

Proof. This is an immediate result of the transformation formula for theta functions (see [5]).

Lemma 2. $\quad \sum_{d} e^{-\pi d^{2} / y^{2}} \log |d|=y \log y+O(y)$ as $y \rightarrow \infty$.

Proof. First, we write

$$
\sum_{d} e^{-\pi d^{2} / y^{2}} \log |d|=\log y \sum_{d} e^{-\pi d^{2} / y^{2}}+\sum_{d} e^{-\pi d^{2} / y^{2}} \log |d / y| .
$$


By Lemma 1,

$$
\log y \sum_{d} e^{-\pi d^{2} / y^{2}}=y \log y+o(\log y)
$$

as $y \rightarrow \infty$. On the other hand, by Riemann-Stieltjes integration,

$$
\begin{aligned}
\sum_{d} e^{-\pi d^{2} / y^{2}} \log |d / y|= & 2 \int_{1^{-}}^{\infty} e^{-\pi u^{2} / y^{2}} \log (u / y) d[u] \\
= & 2 \int_{1}^{\infty} e^{-\pi u^{2} / y^{2}} \log (u / y) d u \\
& -2 \int_{1^{-}}^{\infty} e^{-\pi u^{2} / y^{2}} \log (u / y) d\{u\} .
\end{aligned}
$$

In the first integral, let $v=u / y$; then

$$
\begin{aligned}
2 \int_{1}^{\infty} e^{-\pi u^{2} / y^{2}} \log (u / y) d u & =2 y \int_{y^{-1}}^{\infty} e^{-\pi v^{2}} \log v d v \\
& \leq 2 y \int_{0}^{\infty} e^{-\pi v^{2}} \log v d v \ll y
\end{aligned}
$$

In the second integral we integrate by parts:

$$
\begin{aligned}
-2 \int_{1^{-}}^{\infty} e^{-\pi u^{2} / y^{2}} \log (u / y) d\{u\} & \\
= & {\left[-2\{u\} e^{-\pi u^{2} / y^{2}} \log (u / y)\right]_{1^{-}}^{\infty}+2 \int_{1^{-}}^{\infty}\{u\} d\left(e^{-\pi u^{2} / y^{2}} \log (u / y)\right) } \\
= & 2 e^{-\pi y^{-2}} \log (1 / y)+2 \int_{1^{-}}^{\infty}\{u\} e^{-\pi u^{2} / y^{2}} \frac{d u}{u} \\
& -\frac{4 \pi}{y^{2}} \int_{1^{-}}^{\infty}\{u\} u e^{-\pi u^{2} / y^{2}} \log (u / y) d u \\
= & 2 \int_{y^{-1}}^{\infty}\{v y\} e^{-\pi v^{2}} \frac{d v}{v}-4 \pi \int_{y^{-1}}^{\infty}\{v y\} v e^{-\pi v^{2}} \log v d v+O(\log y) \\
\ll & \log y .
\end{aligned}
$$

This establishes the lemma.

LEMMA 3.

$$
\sum_{d=\square} e^{-\pi d^{2} / y^{2}}=I y^{1 / 2}-1 / 2+O\left(y^{-1 / 2}\right)
$$


as $y \rightarrow \infty$, where $I=(1 / 4) \pi^{-1 / 4} \Gamma(1 / 4)$, and $\sum_{d=\square}$ denotes the sum over those $d$ which are square integers.

P r o of. Using Euler-Maclaurin summation, we have

$$
\begin{aligned}
\sum_{d=\square} e^{-\pi d^{2} / y^{2}}= & \int_{0}^{\infty} e^{-\pi u^{4} / y^{2}} d u-\left[\bar{B}_{1}(u) e^{-\pi u^{4} / y^{2}}\right]_{0}^{\infty} \\
& -\left[\frac{2 \pi}{y^{2}} \bar{B}_{2}(u) u^{3} e^{-\pi u^{4} / y^{2}}\right]_{0}^{\infty} \\
& -\int_{0}^{\infty} \bar{B}_{2}(u)\left(\frac{8 \pi^{2} u^{6}}{y^{4}}-\frac{6 \pi u^{2}}{y^{2}}\right) e^{-\pi u^{4} / y^{2}} d u
\end{aligned}
$$

where $\bar{B}_{1}(u)$ and $\bar{B}_{2}(u)$ are the first and second periodic Bernoulli functions, i.e. $\bar{B}_{1}(u)=\{u\}-1 / 2$ and $\bar{B}_{2}(u)=\{u\}^{2}-\{u\}+1 / 6$. We evaluate the first integral by making the substitution $t=\pi u^{4} / y^{2}$, obtaining

$$
\int_{0}^{\infty} e^{-\pi u^{4} / y^{2}} d u=\frac{1}{4} \pi^{-1 / 4} y^{1 / 2} \int_{0}^{\infty} e^{-t} t^{-3 / 4} d t=\frac{1}{4} \pi^{-1 / 4} \Gamma(1 / 4) y^{1 / 2} .
$$

Next notice that

$$
-\left[\bar{B}_{1}(u) e^{-\pi u^{4} / y^{2}}\right]_{0}^{\infty}=-1 / 2, \quad\left[\frac{2 \pi}{y^{2}} \bar{B}_{2}(u) u^{3} e^{-\pi u^{4} / y^{2}}\right]_{0}^{\infty}=0 .
$$

Finally in the second integral we let $v=u^{4} / y^{2}$ obtaining

$$
\begin{aligned}
\int_{0}^{\infty} \bar{B}_{2}(u) & \left(\frac{8 \pi^{2} u^{6}}{y^{4}}-\frac{6 \pi u^{2}}{y^{2}}\right) e^{-\pi u^{4} / y^{2}} d u \\
& =\int_{0}^{\infty} \bar{B}_{2}\left(y^{1 / 2} v^{1 / 4}\right)\left(\frac{8 \pi^{2}\left(y^{2} v\right)^{3 / 2}}{y^{4}}-\frac{6 \pi\left(y^{2} v\right)^{1 / 2}}{y^{2}}\right) e^{-\pi v} \frac{1}{4} y^{1 / 2} v^{-3 / 4} d v \\
& =O\left(y^{-1 / 2}\right) .
\end{aligned}
$$

This establishes the lemma.

Lemma 4. As $x \rightarrow \infty$,

$$
\sum_{p} a\left(p^{2} / x\right) \log p=\frac{1}{2} K\left(\frac{1}{2}\right) x^{1 / 2}+ \begin{cases}o\left(x^{1 / 2}\right) & \text { unconditionally, } \\ O\left(x^{1 / 4} \log ^{2} x\right) & \text { assuming R.H. }\end{cases}
$$

Here $\sum_{p}$ denotes the sum over primes and R.H. denotes the Riemann Hypothesis.

Proof. Notice that

$$
\sum_{p} a\left(p^{2} / x\right) \log p=\int_{0}^{\infty} a\left(u^{2} / x\right) d \theta(u)
$$


where $\theta(u)=\sum_{p \leq u} \log p$. We write $\theta(u)=u+E(u)$. Then

$$
\int_{0}^{\infty} a\left(u^{2} / x\right) d \theta(u)=\int_{0}^{\infty} a\left(u^{2} / x\right) d u+\int_{0}^{\infty} a\left(u^{2} / x\right) d E(u) .
$$

By letting $v=u^{2} / x$, we get

$$
\int_{0}^{\infty} a\left(u^{2} / x\right) d u=\frac{1}{2} x^{1 / 2} \int_{0}^{\infty} a(v) v^{-1 / 2} d v=\frac{1}{2} K\left(\frac{1}{2}\right) x^{1 / 2} .
$$

On the other hand, recalling that $a(x)$ has compact support in $(0, \infty)$, we get

$$
\begin{aligned}
\int_{0}^{\infty} a\left(u^{2} / x\right) d E(u) & =\left[E(u) a\left(u^{2} / x\right)\right]_{0}^{\infty}-\int_{0}^{\infty} E(u) d a\left(u^{2} / x\right) \\
& =-\frac{2}{x} \int_{0}^{\infty} E(u) a^{\prime}\left(u^{2} / x\right) u d u=-\int_{0}^{\infty} E(\sqrt{x v}) a^{\prime}(v) d v .
\end{aligned}
$$

By the Prime Number Theorem (P.N.T.) we have $E(u)=o(u)$, whence $E(\sqrt{x v})=o(\sqrt{x v})$. Thus,

$$
\int_{0}^{\infty} E(\sqrt{x v}) a^{\prime}(v) d v=o\left(x^{1 / 2} \int_{0}^{\infty} a^{\prime}(v) v^{1 / 2} d v\right)=o\left(x^{1 / 2}\right) .
$$

However, if we assume the Riemann Hypothesis, then $E(u) \ll u^{1 / 2} \log ^{2} u$, in which case

$$
\int_{0}^{\infty} E(\sqrt{x v}) a^{\prime}(v) d v \ll x^{1 / 4} \int_{0}^{\infty} v^{1 / 4} \log ^{2}(x v) a^{\prime}(v) d v \ll x^{1 / 4} \log ^{2} x .
$$

Thus the lemma is established.

LEMMA 5.

$$
\sum_{p} a(p / x) \log p=K(1) x+ \begin{cases}o(x) & \text { unconditionally }, \\ O\left(x^{1 / 2} \log ^{2} x\right) & \text { assuming R.H. }\end{cases}
$$

as $x \rightarrow \infty$.

Proof. We have

$$
\begin{aligned}
\sum_{p} a(p / x) \log p & =\int_{0}^{\infty} a(u / x) d \theta(u) \\
& =\int_{0}^{\infty} a(u / x) d u+\int_{0}^{\infty} a(u / x) d E(u) .
\end{aligned}
$$

On one hand,

$$
\int_{0}^{\infty} a(u / x) d u=x \int_{0}^{\infty} a(v) d v=K(1) x
$$


On the other hand,

$$
\begin{aligned}
\int_{0}^{\infty} a(u / x) d E(u) & =[a(u / x) E(u)]_{0}^{\infty}-\int_{0}^{\infty} E(u) d a(u / x) \\
& =-\frac{1}{x} \int_{0}^{\infty} E(u) a^{\prime}(u / x) d u \\
& =-\int_{0}^{\infty} E(x v) a^{\prime}(v) d v
\end{aligned}
$$

Now, by P.N.T.,

$$
\int_{0}^{\infty} E(x v) a^{\prime}(v) d v=o\left(x \int_{0}^{\infty} v a^{\prime}(v) d v\right)=o(x)
$$

whereas, assuming R.H.,

$$
\int_{0}^{\infty} E(x v) a^{\prime}(v) d v \ll x^{1 / 2} \int_{0}^{\infty} v^{1 / 2} \log ^{2}(x v) a^{\prime}(v) d v \ll x^{1 / 2} \log ^{2} x .
$$

This establishes the lemma.

Lemma 6. As $x \rightarrow \infty$,

$$
\sum_{p} a\left(\frac{p}{x}\right) \frac{\log p}{\sqrt{p}}=K\left(\frac{1}{2}\right) x^{1 / 2}+ \begin{cases}o\left(x^{1 / 2}\right) & \text { unconditionally }, \\ O\left(\log ^{2} x\right) & \text { assuming R.H. }\end{cases}
$$

Proof. First notice that

$$
\begin{aligned}
\sum_{p} a\left(\frac{p}{x}\right) \frac{\log p}{\sqrt{p}} & =\int_{0}^{\infty} a\left(\frac{u}{x}\right) \frac{1}{\sqrt{u}} d \theta(u) \\
& =\int_{0}^{\infty} a\left(\frac{u}{x}\right) \frac{1}{\sqrt{u}} d u+\int_{0}^{\infty} a\left(\frac{u}{x}\right) \frac{1}{\sqrt{u}} d E(u) .
\end{aligned}
$$

On one hand,

$$
\int_{0}^{\infty} a\left(\frac{u}{x}\right) \frac{1}{\sqrt{u}} d u=x^{1 / 2} \int_{0}^{\infty} a(v) \frac{1}{\sqrt{v}} d v=K\left(\frac{1}{2}\right) x^{1 / 2} .
$$

On the other hand,

$$
\begin{aligned}
\int_{0}^{\infty} a\left(\frac{u}{x}\right) \frac{1}{\sqrt{u}} d E(u) & =-\int_{0}^{\infty} E(u) d\left(a\left(\frac{u}{x}\right) u^{-1 / 2}\right) \\
& =\frac{1}{2} \int_{0}^{\infty} E(u) a\left(\frac{u}{x}\right) u^{-3 / 2} d u-\frac{1}{x} \int_{0}^{\infty} E(u) a^{\prime}\left(\frac{u}{x}\right) u^{-1 / 2} d u
\end{aligned}
$$




$$
\begin{aligned}
& =\frac{1}{2} x^{-1 / 2} \int_{0}^{\infty} E(x v) a(v) v^{-3 / 2} d v-x^{-1 / 2} \int_{0}^{\infty} E(x v) a^{\prime}(v) v^{-1 / 2} d v \\
& =o\left(x^{1 / 2}\right) \quad \text { unconditionally, and } \\
& \ll \log ^{2} x \quad \text { assuming R.H. }
\end{aligned}
$$

Thus the lemma is proved.

Lemma 7. As $x \rightarrow \infty$,

$$
\sum_{p} a\left(\frac{p}{x}\right) \frac{\log p}{p}=K(0)+ \begin{cases}o(1) & \text { unconditionally } \\ O\left(x^{-1 / 2} \log ^{2} x\right) & \text { assuming R.H. }\end{cases}
$$

Proof. First notice that

$$
\begin{aligned}
\sum_{p} a\left(\frac{p}{x}\right) \frac{\log p}{p} & =\int_{0}^{\infty} a\left(\frac{u}{x}\right) \frac{1}{u} d \theta(u) \\
& =\int_{0}^{\infty} a\left(\frac{u}{x}\right) \frac{1}{u} d u+\int_{0}^{\infty} a\left(\frac{u}{x}\right) \frac{1}{u} d E(u) .
\end{aligned}
$$

Now,

$$
\int_{0}^{\infty} a\left(\frac{u}{x}\right) \frac{1}{u} d u=\int_{0}^{\infty} a(v) \frac{d v}{v}=K(0)
$$

Also,

$$
\begin{aligned}
& \int_{0}^{\infty} a\left(\frac{u}{x}\right) \frac{1}{u} d E(u)=-\int_{0}^{\infty} E(u) d\left(a\left(\frac{u}{x}\right) u^{-1}\right) \\
& =\int_{0}^{\infty} E(u) a\left(\frac{u}{x}\right) u^{-2} d u-\frac{1}{x} \int_{0}^{\infty} E(u) a^{\prime}\left(\frac{u}{x}\right) u^{-1} d u \\
& =\frac{1}{x} \int_{0}^{\infty} E(x v) a(v) v^{-2} d v-\frac{1}{x} \int_{0}^{\infty} E(x v) a^{\prime}(v) v^{-1} d v \\
& =o(1) \quad \text { unconditionally, and } \\
& =O\left(x^{-1 / 2} \log ^{2} x\right) \quad \text { under R.H. }
\end{aligned}
$$

This establishes the lemma.

Lemma 8. As $x \rightarrow \infty$,

$$
\sum_{p} a\left(\frac{p^{2}}{x}\right) \frac{\log p}{p}=\frac{1}{2} K(0)+ \begin{cases}o(1) & \text { unconditionally } \\ O\left(x^{-1 / 4} \log ^{2} x\right) & \text { assuming R.H. }\end{cases}
$$


Pr o of. Notice that

$$
\begin{aligned}
\sum_{p} a\left(\frac{p^{2}}{x}\right) \frac{\log p}{p} & =\int_{0}^{\infty} a\left(\frac{u^{2}}{x}\right) \frac{1}{u} d \theta(u) \\
& =\int_{0}^{\infty} a\left(\frac{u^{2}}{x}\right) \frac{1}{u} d u+\int_{0}^{\infty} a\left(\frac{u^{2}}{x}\right) \frac{1}{u} d E(u) .
\end{aligned}
$$

But then

$$
\int_{0}^{\infty} a\left(\frac{u^{2}}{x}\right) \frac{1}{u} d u=\frac{1}{2} \int_{0}^{\infty} a(v) \frac{d v}{v}=\frac{1}{2} K(0) .
$$

On the other hand,

$$
\begin{aligned}
\int_{0}^{\infty} a\left(\frac{u^{2}}{x}\right) \frac{1}{u} d E(u)= & -\int_{0}^{\infty} E(u) d\left(a\left(\frac{u^{2}}{x}\right) u^{-1}\right) \\
= & \int_{0}^{\infty} E(u) a\left(\frac{u^{2}}{x}\right) u^{-2} d u-\frac{2}{x} \int_{0}^{\infty} E(u) a^{\prime}\left(\frac{u^{2}}{x}\right) d u \\
= & \frac{1}{2} x^{-1 / 2} \int_{0}^{\infty} E(\sqrt{x v}) a(v) v^{-3 / 2} d v \\
& -x^{-1 / 2} \int_{0}^{\infty} E(\sqrt{x v}) a^{\prime}(v) v^{-1 / 2} d v \\
= & o(1) \quad \text { unconditionally, and } \\
& \ll x^{-1 / 4} \log ^{2} x \quad \text { assuming R.H. }
\end{aligned}
$$

Thus the lemma follows.

Lemma 9. As $x \rightarrow \infty$,

$$
\sum_{p} a(p / x) p^{1 / 2} \log ^{2} p \ll x^{3 / 2} \log x .
$$

Proof. We have

$$
\begin{aligned}
\sum_{p} a(p / x) p^{1 / 2} \log ^{2} p= & \int_{0}^{\infty} a(u / x) u^{1 / 2} \log (u) d \theta(u) \\
= & \int_{0}^{\infty} a(u / x) u^{1 / 2} \log (u) d u \\
& +\int_{0}^{\infty} a(u / x) u^{1 / 2} \log (u) d E(u) .
\end{aligned}
$$


On one hand,

$$
\int_{0}^{\infty} a(u / x) u^{1 / 2} \log (u) d u=x^{3 / 2} \int_{0}^{\infty} a(v) v^{1 / 2} \log (x v) d v \ll x^{3 / 2} \log x .
$$

On the other hand,

$$
\begin{aligned}
\int_{0}^{\infty} a(u / x) u^{1 / 2} \log (u) d E(u)= & -\int_{0}^{\infty} E(u) d\left(a(u / x) u^{1 / 2} \log u\right) \\
= & -\frac{1}{x} \int_{0}^{\infty} E(u) a^{\prime}(u / x) u^{1 / 2} \log (u) d u \\
& -\frac{1}{2} \int_{0}^{\infty} E(u) a(u / x) u^{-1 / 2} \log (u) d u \\
& -\int_{0}^{\infty} E(u) a(u / x) u^{-1 / 2} d u \\
= & -x^{1 / 2} \int_{0}^{\infty} E(x v) a^{\prime}(v) v^{1 / 2} \log (x v) d v \\
& -\frac{1}{2} x^{1 / 2} \int_{0}^{\infty} E(x v) a(v) v^{-1 / 2} \log (x v) d v \\
& -x^{1 / 2} \int_{0}^{\infty} E(x v) a(v) v^{-1 / 2} d v \\
\ll & x^{3 / 2} \log x .
\end{aligned}
$$

This establishes the lemma.

3. The main theorem. Let $x$ and $D$ be positive real numbers. We are interested in the distribution of the nontrivial zeros, $\varrho=\beta+\gamma i$ with $\gamma$ small, of the $L$-series, $L\left(s, \chi_{d}\right)$, where $\chi_{d}$ denotes the Kronecker symbol $(d / \cdot)$. More specifically, we consider the expression

$$
\sum_{d} e^{-\pi d^{2} / D^{2}} \sum_{\varrho(d)} K(\varrho) x^{\varrho} .
$$

The inner sum is over all the nontrivial zeros of $L\left(s, \chi_{d}\right)$ and the other sum is over all nonzero integers. In essence we are averaging $\sum_{\varrho(d)} K(\varrho) x^{\varrho}$ over those $d$ which are in absolute value $\leq D$. For technical reasons we use the weighting $\sum_{d} e^{-\pi d^{2} / D^{2}}$ instead of $\sum_{|d| \leq D}$, which simplifies the calculations at one point in the proof of the main theorem below where the Poisson summation formula is applied. 
Theorem. If $x=o(D)$, then as $x \rightarrow \infty$,

$$
\begin{aligned}
\sum_{d} e^{-\pi d^{2} / D^{2}} & \sum_{\varrho(d)} K(\varrho) x^{\varrho} \\
= & -\frac{1}{2} K\left(\frac{1}{2}\right) D x^{1 / 2}+I K(1) x D^{1 / 2}+a(1 / x) D \log D \\
& +o\left(D x^{1 / 2}\right)+O(\min (x D, D \log D \log x))+O\left(x^{3 / 2} \log x\right) .
\end{aligned}
$$

Assume the Generalized Riemann Hypothesis (G.R.H.). Then as $x, D \rightarrow \infty$,

$$
\begin{aligned}
& \sum_{d} e^{-\pi d^{2} / D^{2}} \sum_{\varrho(d)} K(\varrho) x^{\varrho} \\
& \quad=\left\{\begin{array}{cl}
-\frac{1}{2} K\left(\frac{1}{2}\right) D x^{1 / 2}+I K(1) x D^{1 / 2}+a(1 / x) D \log D & \\
+O\left(D x^{1 / 3} \log x\right)+O(\min (x D, D \log D \log x)) & \text { if } x=o(D), \\
+O\left(x^{3 / 2} \log x\right) & \text { if } D=o(x) . \\
O\left(x \log ^{2} x\right)+O\left(D x^{1 / 3} \log ^{2} x\right)+O\left(D^{3 / 2}\right) & O(\min (x)
\end{array}\right.
\end{aligned}
$$

The statements are also true for $x=1$, provided we replace $O(\min (x D$, $D \log D \log x)$ ) by $O(D)$.

In all the statements the implied constants depend only on $K(s)$.

Proof. We use the explicit formula

$$
\begin{aligned}
\sum_{\varrho(d)} K(\varrho) x^{\varrho}= & K(1) E\left(\chi_{d}\right) x-\sum_{n=1}^{\infty} a(n / x) \Lambda(n)(d / n) \\
& +a(1 / x) \log (|d| / \pi)+O(\min (x, \log |d| \log x)) .
\end{aligned}
$$

See, for example, [8]. Here $E(\chi)=1$ or 0 according as $\chi$ is principal or not. Then

$$
\sum_{d} e^{-\pi d^{2} / D^{2}} \sum_{\varrho(d)} K(\varrho) x^{\varrho}=A+B+C+O
$$

where

$$
\begin{aligned}
A & =K(1) x \sum_{d} E\left(\chi_{d}\right) e^{-\pi d^{2} / D^{2}}, \\
B & =-\sum_{n=1}^{\infty} a(n / x) \Lambda(n) \sum_{d} e^{-\pi d^{2} / D^{2}}(d / n) \\
C & =a(1 / x) \sum_{d} e^{-\pi d^{2} / D^{2}} \log (|d| / \pi) \\
O & =O\left(\min \left(x \sum_{d} e^{-\pi d^{2} / D^{2}}, \log x \sum_{d} e^{-\pi d^{2} / D^{2}} \log |d|\right)\right) .
\end{aligned}
$$

By Lemmas 1 and $2, O=O(\min (x D, D \log D \log x))$ as $D \rightarrow \infty$. By 
Lemma 3,

$$
A=K(1) x \sum_{d=\square} e^{-\pi d^{2} / D^{2}}=I K(1) x D^{1 / 2}-\frac{1}{2} K(1) x+O\left(x D^{-1 / 2}\right),
$$

as $D \rightarrow \infty$. By Lemma 2 ,

$$
C=a(1 / x) D \log D+O(D)
$$

as $D \rightarrow \infty$.

Now consider $B$. We decompose $B$ as $B=B_{1}+B_{2}+B_{3}+B_{4}$ where

$$
\begin{aligned}
& B_{1}=-\sum_{p} a(p / x) \log p \sum_{d} e^{-\pi d^{2} / D^{2}}(d / p), \\
& B_{2}=-\sum_{p} a\left(p^{2} / x\right) \log p \sum_{d} e^{-\pi d^{2} / D^{2}}, \\
& B_{3}=\sum_{p} a\left(p^{2} / x\right) \log p \sum_{d, p \mid d} e^{-\pi d^{2} / D^{2}}, \\
& B_{4}=-\sum_{p^{m}, m \geq 3} a\left(p^{m} / x\right) \log p \sum_{d} e^{-\pi d^{2} / D^{2}}\left(d / p^{m}\right) .
\end{aligned}
$$

Since $a\left(p^{m} / x\right)=0$ unless $p^{m} / x \in[A, B]$, and by Lemma 1 , we see

$$
\begin{aligned}
B_{4} & \ll \sum_{p^{m}, m \geq 3}\left|a\left(p^{m} / x\right)\right| \log p \sum_{d} e^{-\pi d^{2} / D^{2}} \\
& \ll D \sum_{p^{m}, m \geq 3}\left|a\left(p^{m} / x\right)\right| \log p \ll D x^{1 / 3} \log x .
\end{aligned}
$$

Next, notice that

$$
\begin{aligned}
B_{3} & =\sum_{p} a\left(p^{2} / x\right) \log p \sum_{m} e^{-\pi m^{2} p^{2} / D^{2}}=\sum_{p} a\left(p^{2} / x\right) \log p(D / p+o(1)) \\
& =O(D)+O\left(x^{1 / 2}\right)
\end{aligned}
$$

by Lemmas 1,4 , and 8 .

By Lemmas 1 and 4,

$$
\begin{aligned}
B_{2} & =\left(-\frac{1}{2} K\left(\frac{1}{2}\right) x^{1 / 2}+O\left(x^{1 / 4} \log ^{2} x\right)\right)(D+o(1)) \\
& =-\frac{1}{2} K\left(\frac{1}{2}\right) D x^{1 / 2}+O\left(x^{1 / 2}\right)+O\left(D x^{1 / 4} \log ^{2} x\right)
\end{aligned}
$$

assuming R.H., while

$$
\begin{aligned}
B_{2} & =\left(-\frac{1}{2} K\left(\frac{1}{2}\right) x^{1 / 2}+o\left(x^{1 / 2}\right)\right)(D+o(1)) \\
& =-\frac{1}{2} K\left(\frac{1}{2}\right) D x^{1 / 2}+O\left(x^{1 / 2}\right)+o\left(D x^{1 / 2}\right)
\end{aligned}
$$

unconditionally.

Finally we consider $B_{1}$. We consider two cases. 
Case 1: $x=o(D)$. We use the Pólya-Vinogradov bound on character sums as follows:

$$
\begin{aligned}
\sum_{d} e^{-\pi d^{2} / D^{2}}(d / p) & \ll\left|\int_{0}^{\infty} e^{-\pi u^{2} / D^{2}} d\left(\sum_{d \leq u}(d / p)\right)\right| \\
& =\left|\int_{0}^{\infty} \sum_{d \leq u}(d / p) d\left(e^{-\pi u^{2} / D^{2}}\right)\right| \\
& \ll D^{-2} p^{1 / 2} \log p \int_{0}^{\infty} u e^{-\pi u^{2} / D^{2}} d u \\
& =p^{1 / 2} \log p \int_{0}^{\infty} v e^{-\pi v^{2}} d v \ll p^{1 / 2} \log p .
\end{aligned}
$$

But then by Lemma 9,

$$
B_{1} \ll \sum_{p} a(p / x) p^{1 / 2} \log ^{2} p \ll x^{3 / 2} \log x .
$$

CASE 2: $D=o(x)$. In this case we assume G.R.H. By the transformation formula for theta functions (see e.g. [5]),

$$
B_{1}=-\sum_{p} a\left(\frac{p}{x}\right) \log p \frac{D}{\sqrt{p}} \sum_{m}\left(\frac{m}{p}\right) e^{-\pi m^{2} D^{2} / p^{2}} .
$$

We now write $B_{1}=B_{11}+B_{12}+B_{13}$, where

$$
\begin{aligned}
& B_{11}=-D \sum_{p} a\left(\frac{p}{x}\right) \frac{\log p}{\sqrt{p}} \sum_{m=\square} e^{-\pi m^{2} D^{2} / p^{2}}, \\
& B_{12}=D \sum_{p} a\left(\frac{p}{x}\right) \frac{\log p}{\sqrt{p}} \sum_{\substack{m=\square \\
p \mid m}} e^{-\pi m^{2} D^{2} / p^{2}}, \\
& B_{13}=-D \sum_{p} a\left(\frac{p}{x}\right) \frac{\log p}{\sqrt{p}} \sum_{m \neq \square}\left(\frac{m}{p}\right) e^{-\pi m^{2} D^{2} / p^{2}} .
\end{aligned}
$$

By Lemmas 3, 5, 6, and 7 (and assuming R.H.),

$$
\begin{aligned}
B_{11}= & -D \sum_{p} a\left(\frac{p}{x}\right) \frac{\log p}{\sqrt{p}}\left(\sqrt{\frac{p}{D}} I-\frac{1}{2}+O(\sqrt{D / p})\right) \\
= & -I D^{1 / 2} \sum_{p} a\left(\frac{p}{x}\right) \log p+\frac{1}{2} D \sum_{p} a\left(\frac{p}{x}\right) \frac{\log p}{\sqrt{p}} \\
& +O\left(D^{3 / 2} \sum_{p} a\left(\frac{p}{x}\right) \frac{\log p}{p}\right)
\end{aligned}
$$




$$
\begin{aligned}
= & -I K(1) D^{1 / 2} x+\frac{1}{2} K\left(\frac{1}{2}\right) D x^{1 / 2}+O\left(D^{1 / 2} x^{1 / 2} \log ^{2} x\right) \\
& +O\left(D \log ^{2} x\right)+O\left(D^{3 / 2}\right) .
\end{aligned}
$$

Next, we have

$$
\begin{aligned}
B_{12} & =D \sum_{p} a\left(\frac{p}{x}\right) \frac{\log p}{\sqrt{p}} \sum_{m=1}^{\infty} e^{-\pi m^{4} p^{2} D^{2}} \\
& \ll\left|D \sum_{p} a\left(\frac{p}{x}\right) \frac{\log p}{\sqrt{p}} \cdot \frac{1}{\sqrt{p D}}\right| \ll D^{1 / 2} .
\end{aligned}
$$

Finally, we consider $B_{13}$. Since $a(u)=0$ if $u>B$, we see

$$
B_{13} \ll D \sum_{m \neq \square} e^{-\pi m^{2} D^{2} /\left(B^{2} x^{2}\right)}\left|\sum_{p} a\left(\frac{p}{x}\right) \frac{\log p}{\sqrt{p}}\left(\frac{m}{p}\right)\right| .
$$

We claim that, under G.R.H.,

$$
\sum_{p} a\left(\frac{p}{x}\right) \frac{\log p}{\sqrt{p}}\left(\frac{m}{p}\right) \ll \log ^{2} m x
$$

for $m \ll x$. For,

$$
\begin{aligned}
\sum_{p} a\left(\frac{p}{x}\right) \frac{\log p}{\sqrt{p}}\left(\frac{m}{p}\right)= & \int_{0}^{\infty} a\left(\frac{u}{x}\right) \frac{1}{\sqrt{u}} d\left(\sum_{p \leq u}\left(\frac{m}{p}\right) \log p\right) \\
= & -\int_{0}^{\infty} \sum_{p \leq u}(m / p) \log p d\left(a(u / x) u^{-1 / 2}\right) \\
= & \frac{1}{2} \int_{0}^{\infty} \sum_{p \leq u}(m / p) \log p a(u / x) u^{-3 / 2} d u \\
& -\frac{1}{x} \int_{0}^{\infty} \sum_{p \leq u}^{\infty}(m / p) \log p a^{\prime}(u / x) u^{-1 / 2} d u \\
= & \frac{1}{2} x^{-1 / 2} \int_{0}^{\infty} \sum_{p \leq x v}(m / p) \log p a(v) v^{-3 / 2} d v \\
& -x^{-1 / 2} \int_{0}^{\infty} \sum_{p \leq x v}(m / p) \log p a^{\prime}(v) v^{-1 / 2} d v
\end{aligned}
$$

$\ll \log ^{2} m x$,

since under G.R.H.

$$
\sum_{p \leq x}(m / p) \log p \ll x^{1 / 2} \log ^{2} m x
$$


for $m \ll x$, as readily follows as in [5]. But then

$$
B_{13} \ll D \sum_{m} e^{-\pi m^{2} D^{2} /\left(B^{2} x^{2}\right)} \log ^{2} m x \ll x \log ^{2}\left(x^{2} / D\right) .
$$

This proves the main statements of the Theorem. The case where $x=1$ follows immediately from the identity

$$
\sum_{\varrho(d)} K(\varrho)=a(1) \log |d|+O(1) \quad \text { as }|d| \rightarrow \infty .
$$

The Theorem is now established.

We now set $x=D^{\alpha}$ and define

$$
F_{K}(\alpha, D)=D^{-1} \sum_{d \neq 0} e^{-\pi d^{2} / D^{2}}\left(\frac{1}{2} K\left(\frac{1}{2}\right)\right)^{-1} \sum_{\varrho} K(\varrho) D^{i \alpha \gamma} .
$$

Then as an immediate corollary to the Theorem, we have

Corollary 1. Assume G.R.H. Then as $D \rightarrow \infty$,

$$
\begin{aligned}
& F_{K}(\alpha, D) \\
& \quad= \begin{cases}-1+\left(\frac{1}{2} K\left(\frac{1}{2}\right)\right)^{-1} D^{-\alpha / 2} a\left(D^{-\alpha}\right) \log D+o(1) & \text { if }|\alpha|<1, \\
0+o(1) & \text { if } 1<|\alpha|<2 .\end{cases}
\end{aligned}
$$

The implied constants depend only on the kernel $K$.

Notice that we obtained information about $F_{K}$ for $|\alpha|<2$ only, even assuming G.R.H., because of our very rough estimate on the sum $B_{3}$ in the proof of the Theorem. However, we would expect that $F_{K}(\alpha, D)=o(1)$ for all $|\alpha|>1$.

The Theorem remains valid if $K(s)$ is replaced by the usual weighting $K(s)=1 / s$, in which case $a(x)=\xi_{[0,1]}(x)$, the characteristic function of the unit interval. The only changes in the proof of the Theorem occur in the error estimates in Lemmas 7 and 8, which do not affect the results in the statement of the Theorem. If we assume G.R.H. and divide by $x^{1 / 2}$, the Theorem then takes the following form:

$$
\begin{aligned}
& \sum_{d} e^{-\pi d^{2} / D^{2}} \sum_{\varrho(d)} \frac{x^{i \gamma}}{\varrho} \\
& = \begin{cases}-D+I x^{1 / 2} D^{1 / 2}+x^{-1 / 2} D \log D+O\left(x \log ^{2} x\right) & \\
+O\left(D x^{-1 / 6} \log x\right)+O\left(\min \left(x^{1 / 2} D, x^{-1 / 2} D \log D \log x\right)\right) & \text { if } x=o(D), \\
O\left(x^{1 / 2} \log ^{2} x\right)+O\left(D x^{-1 / 6} \log x\right)+O\left(D^{3 / 2} x^{-1 / 2}\right) & \text { if } D=o(x) .\end{cases}
\end{aligned}
$$

Since $\sum_{d} \exp \left(-\pi d^{2} / D^{2}\right)$ is asymptotic to $D$, we see, roughly speaking, that on average (over $d$ ), 


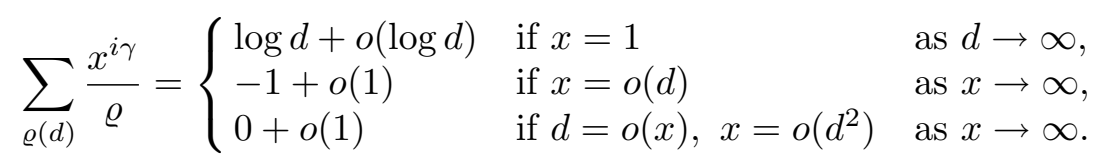

By examining the proof of the Theorem we see that

$$
\begin{aligned}
D^{-1} x^{-1 / 2} \sum_{d \neq \square} e^{-\pi d^{2} / D^{2}} \sum_{p^{2} \leq x} \log p+D^{-1} x^{-1 / 2} \sum_{d \neq \square} e^{-\pi d^{2} / D^{2}} \sum_{p \leq x}(d / p) \log p \\
= \begin{cases}1+o(1) & \text { if } x=o(D), \\
0+o(1) & \text { if } D=o(x), x=o\left(D^{2}\right),\end{cases}
\end{aligned}
$$

as $x \rightarrow \infty$. But

$$
D^{-1} x^{-1 / 2} \sum_{d \neq \square} e^{-\pi d^{2} / D^{2}} \sum_{p^{2} \leq x} \log p=1+o(1)
$$

as $D, x \rightarrow \infty$. Thus by our Theorem,

$$
\begin{aligned}
D^{-1} x^{-1 / 2} \sum_{d \neq \square} e^{-\pi d^{2} / D^{2}} \sum_{p \leq x}(d / p) \log p \\
\quad= \begin{cases}0+o(1) & \text { if } x=o(D), \\
-1+o(1) & \text { if } D=o(x), x=o\left(D^{2}\right),\end{cases}
\end{aligned}
$$

as $x \rightarrow \infty$. This shows that, roughly speaking, on the average over $d \neq \square$, there is a preponderance of primes $\leq x$ which are quadratic nonresidues $\bmod d$ for $x$ between $d$ and $d^{2}$ (probably all $x=d^{\alpha}, \alpha \geq 2$ fixed, based on our predicted behavior of $F_{K}(\alpha)$ for $\left.|\alpha|>2\right)$; whereas for $x$ less than $d$ the residues and nonresidues mod $d$ are more or less evenly distributed.

4. Applications. We now investigate the distribution of the nontrivial zeros of $L\left(s, \chi_{d}\right)$ which are close to the real axis.

Corollary 2. Suppose $r(\alpha)$ is an even function defined on $(-\infty, \infty)$ such that its Fourier transform,

$$
\widehat{r}(\alpha)=\int_{-\infty}^{\infty} r(u) e^{-2 \pi i \alpha u} d u
$$

exists and has support in $[-2,2]$ and that $\int_{-\infty}^{\infty} \alpha r(\alpha) d \alpha$ converges. Then under G.R.H., and as $D \rightarrow \infty$,

$$
\begin{aligned}
D^{-1} \sum_{d} e^{-\pi d^{2} / D^{2}}\left(\frac{1}{2} K\left(\frac{1}{2}\right)\right)^{-1} & \sum_{\varrho(d)} K(\varrho) r\left(\frac{\gamma \log D}{2 \pi}\right) \\
& =2 \int_{-\infty}^{\infty}\left(1-\frac{\sin 2 \pi \alpha}{2 \pi \alpha}\right) r(\alpha) d \alpha+o(1),
\end{aligned}
$$

where the implied constant depends only on the kernel $K$. 
Proof. By Corollary 1 and since $\widehat{r}(\alpha)$ is supported in $[-2,2]$,

$$
\begin{aligned}
& \int_{-\infty}^{\infty} F_{K}(\alpha, D) \widehat{r}(\alpha) d \alpha \\
& \quad=\int_{-\infty}^{\infty}\left(-\xi_{[-1,1]}(\alpha)+\left(\frac{1}{2} K\left(\frac{1}{2}\right)\right)^{-1} D^{-\alpha / 2} a\left(D^{-\alpha}\right) \log D\right) \widehat{r}(\alpha) d \alpha+o(1),
\end{aligned}
$$

where $\xi_{[-1,1]}$ is the characteristic function of $[-1,1]$. But

$$
\int_{-\infty}^{\infty} \xi_{[-1,1]}(\alpha) \widehat{r}(\alpha) d \alpha=\int_{-\infty}^{\infty} \widehat{\xi}_{[-1,1]}(\alpha) r(\alpha) d \alpha=2 \int_{-\infty}^{\infty} \frac{\sin 2 \pi \alpha}{2 \pi \alpha} r(\alpha) d \alpha .
$$

On the other hand,

$$
\int_{-\infty}^{\infty} D^{-\alpha / 2} a\left(D^{-\alpha}\right) \widehat{r}(\alpha) d \alpha=\int_{-\infty}^{\infty}\left(D^{-\alpha / 2} a\left(D^{-\alpha}\right)\right)^{\wedge} r(\alpha) d \alpha .
$$

But

$$
\left(D^{-\alpha / 2} a\left(D^{-\alpha}\right)\right)^{\wedge}=\int_{-\infty}^{\infty} D^{-\beta / 2} a\left(D^{-\beta}\right) e^{-2 \pi i \alpha \beta} d \beta .
$$

By a change of variable $t=D^{-\beta}$, this last integral equals

$$
\begin{aligned}
& \frac{1}{\log D} \int_{0}^{\infty} a(t) t^{1 / 2+2 \pi i \alpha / \log D} \frac{d t}{t} \\
& \quad=\frac{1}{\log D} \int_{0}^{\infty} a(t) t^{1 / 2} \frac{d t}{t}+\frac{1}{\log D} \int_{0}^{\infty} a(t) t^{1 / 2}\left(t^{2 \pi i \alpha / \log D}-1\right) \frac{d t}{t} .
\end{aligned}
$$

Notice that

$$
\begin{aligned}
t^{2 \pi i \alpha / \log D}-1 & =\exp \left(2 \pi i \frac{\log t}{\log D} \alpha\right)-1 \\
& =2 \pi i \alpha \frac{\log t}{\log D} \exp \left(2 \pi i \frac{\log t}{\log D} \theta_{\alpha}\right)
\end{aligned}
$$

for some $\theta_{\alpha}$ between 0 and $\alpha$. Therefore

$$
t^{2 \pi i \alpha / \log D}-1 \ll \frac{\log t}{\log D} \alpha,
$$

so that

$$
\frac{1}{\log D} \int_{0}^{\infty} a(t) t^{1 / 2}\left(t^{2 \pi i \alpha / \log D}-1\right) \frac{d t}{t} \ll \frac{\alpha}{\log ^{2} D}
$$

where the implied constant is independent of $\alpha$ and $D$. Therefore, 


$$
\begin{aligned}
\int_{-\infty}^{\infty}\left(D^{-\alpha / 2} a\left(D^{-\alpha}\right)\right)^{\wedge} r(\alpha) d \alpha & \\
& =\frac{1}{\log D} K\left(\frac{1}{2}\right) \int_{-\infty}^{\infty} r(\alpha) d \alpha+O\left(\frac{1}{\log ^{2} D} \int_{-\infty}^{\infty} \alpha r(\alpha) d \alpha\right) \\
& =\frac{1}{\log D} K\left(\frac{1}{2}\right) \int_{-\infty}^{\infty} r(\alpha) d \alpha+O\left(\frac{1}{\log ^{2} D}\right)
\end{aligned}
$$

Thus,

$$
\begin{aligned}
\int_{-\infty}^{\infty}\left(-\xi_{[-1,1]}(\alpha)+\left(\frac{1}{2} K\left(\frac{1}{2}\right)\right)^{-1} D^{-\alpha / 2} a\left(D^{-\alpha}\right) \log D\right) \widehat{r}(\alpha) d \alpha \\
=2 \int_{-\infty}^{\infty}\left(1-\frac{\sin 2 \pi \alpha}{2 \pi \alpha}\right) r(\alpha) d \alpha+O\left(\frac{1}{\log D}\right) .
\end{aligned}
$$

Consequently,

$$
\int_{-\infty}^{\infty} F_{K}(\alpha, D) \widehat{r}(\alpha) d \alpha=2 \int_{-\infty}^{\infty}\left(1-\frac{\sin 2 \pi \alpha}{2 \pi \alpha}\right) r(\alpha) d \alpha+o(1)
$$

On the other hand,

$$
\begin{aligned}
\int_{-\infty}^{\infty} F_{K}(\alpha, D) \widehat{r}(\alpha) d \alpha & \\
= & \left(\frac{1}{2} K\left(\frac{1}{2}\right) D\right)^{-1} \sum_{d} e^{-\pi d^{2} / D^{2}} \sum_{\varrho(d)} K(\varrho) \int_{-\infty}^{\infty} e^{i \alpha \gamma \log D} \widehat{r}(\alpha) d \alpha \\
& =\left(\frac{1}{2} K\left(\frac{1}{2}\right) D\right)^{-1} \sum_{d} e^{-\pi d^{2} / D^{2}} \sum_{\varrho(d)} K(\varrho) r\left(\frac{\gamma \log D}{2 \pi}\right),
\end{aligned}
$$

since $\widehat{\widehat{r}}(\alpha)=r(-\alpha)=r(\alpha)$. This establishes the corollary.

If we had considered the nontrivial zeros of all Dirichlet $L$-functions, then $(1-\sin 2 \pi \alpha) /(2 \pi \alpha)$ in the corollary would have been replaced by 1 . Thus for quadratic $L$-functions, the nontrivial zeros near the real axis are sparser than on the average so that in some sense they are "being repelled" from the real axis.

The next application gives an upper bound on the number of $d$ 's for which $L\left(1 / 2, \chi_{d}\right)=0$.

Corollary 3. Suppose $1 / 2$ is a "zero" of $L\left(s, \chi_{d}\right)$ of multiplicity $m_{d}$ $\geq$ 0. Assuming G.R.H.,

$$
\sum_{d} e^{-\pi d^{2} / D^{2}} m_{d} \leq \frac{1}{8} D+O\left(\frac{D}{\log D}\right) \quad \text { as } D \rightarrow \infty .
$$


Proof. Choose a kernel $K$ with $\Re(K(1 / 2+i t)) \geq 0$. Let $\lambda \in(1,2]$ and $r(u)=(\sin \pi \lambda u /(\pi \lambda u))^{2}$. A direct computation shows that $\widehat{r}(\alpha)=$ $\lambda^{-2} \max (\lambda-|\alpha|, 0)$. From the proof of Corollary 2,

$$
\left(\frac{1}{2} K\left(\frac{1}{2}\right) D\right)^{-1} \sum_{d} e^{-\pi d^{2} / D^{2}} \sum_{\varrho(d)} K(\varrho) r\left(\frac{\gamma \log D}{2 \pi}\right)=\int_{-\infty}^{\infty} F_{K}(\alpha, D) \widehat{r}(\alpha) d \alpha .
$$

By Corollary 1, we have

$$
\begin{aligned}
& \int_{-\infty}^{\infty} F_{K}(\alpha, D) \widehat{r}(\alpha) d \alpha \\
& =\int_{-\infty}^{\infty}\left(-\xi_{[-1,1]}(\alpha)+\left(\frac{1}{2} K\left(\frac{1}{2}\right)\right)^{-1} D^{-\alpha / 2} a\left(D^{-\alpha}\right) \log D\right) \widehat{r}(\alpha) d \alpha+o(1) \\
& =-\frac{1}{\lambda^{2}} \int_{-1}^{1}(\lambda-|\alpha|) d \alpha+\left(\frac{1}{2} K\left(\frac{1}{2}\right)\right)^{-1} \log D \int_{-\infty}^{\infty} D^{-\alpha / 2} a\left(D^{-\alpha}\right) \widehat{r}(\alpha) d \alpha+o(1) \\
& =-\frac{2}{\lambda^{2}}\left[\lambda \alpha-\frac{\alpha^{2}}{2}\right]_{0}^{1}+\left(\frac{1}{2} K\left(\frac{1}{2}\right)\right)^{-1} \log D \frac{K(1 / 2)}{\log D} \int_{-\infty}^{\infty} r(\alpha) d \alpha+O\left(\frac{1}{\log D}\right) \\
& =\frac{1}{\lambda^{2}}-\frac{2}{\lambda}+2 \widehat{r}(0)+O\left(\frac{1}{\log D}\right)=\frac{1}{\lambda^{2}}+O\left(\frac{1}{\log D}\right) .
\end{aligned}
$$

On the other hand,

$$
\begin{aligned}
& \int_{-\infty}^{\infty} F_{K}(\alpha, D) \widehat{r}(\alpha) d \alpha \\
&=\left(\frac{1}{2} K\left(\frac{1}{2}\right) D\right)^{-1} \sum_{d} e^{-\pi d^{2} / D^{2}} \sum_{\varrho(d)} K(\varrho)\left(\frac{\sin \left(\frac{\lambda}{2} \gamma \log D\right)}{\frac{\lambda}{2} \gamma \log D}\right)^{2} .
\end{aligned}
$$

Therefore,

$$
\begin{aligned}
\frac{2}{D} \sum_{d} e^{-\pi d^{2} / D^{2}} m_{d} & \\
& =\left(\frac{1}{2} K\left(\frac{1}{2}\right) D\right)^{-1} \sum_{d} e^{-\pi d^{2} / D^{2}} \sum_{\substack{\varrho(d) \\
\varrho=1 / 2}} K(\varrho) \\
& \leq\left(\frac{1}{2} K\left(\frac{1}{2}\right) D\right)^{-1} \sum_{d} e^{-\pi d^{2} / D^{2}} \sum_{\varrho(d)} K(\varrho)\left(\frac{\sin \left(\frac{\lambda}{2} \gamma \log D\right)}{\frac{\lambda}{2} \gamma \log D}\right)^{2} \\
& =\frac{1}{\lambda^{2}}+O\left(\frac{1}{\log D}\right) .
\end{aligned}
$$


By taking $\lambda=2$, we have

$$
\frac{2}{D} \sum_{d} e^{-\pi d^{2} / D^{2}} m_{d} \leq \frac{1}{4}+O\left(\frac{1}{\log D}\right) .
$$

Multiplying by $D / 2$ establishes the corollary.

REMARK. Since $m_{d}$ is always even, this corollary implies that $L\left(1 / 2, \chi_{d}\right)$ $=0$ for at most $1 / 16$ of all $d$ 's.

Acknowledgements. We would like to thank Professor Hugh L. Montgomery for bringing this problem to our attention.

Added in proof. Since the acceptance for publication of this paper, the BAMS article of N. Katz and P. Sarnak appeared in Bull. Amer. Math. Soc. 36 (1999), 1-26. Their article contains the statement of our Corollary 2. (A proof different from ours may be found in the appendix of an early unpublished version of their BAMS article.)

Several months later, we had communication with Peter Sarnak in which he offered some input concerning our article. We would like to thank Professor Sarnak for reading our manuscript and offering improvements which we have tried to realize in this article's final form.

Also, it has been brought to our attention that Sarnak's Ph.D. student K. Soundararajan has proven unconditionally that $L\left(1 / 2, \chi_{d}\right)=0$ for at most $1 / 8$ of all $d$ 's.

\section{References}

[1] H.-J. Bentz, Discrepancies in the distribution of prime numbers, J. Number Theory 15 (1982), 252-274.

[2] H.-J. Bentz and J. Pintz, Quadratic residues and the distribution of prime numbers, Monatsh. Math. 90 (1982), 91-100.

[3] H.-J. Besenfelder, Die "Weilsche explizite Formel" und temperierte Distributionen, J. Reine Angew. Math. 293/294 (1977), 228-257.

[4] P. L. Chebyshev, Lettres de M. le professeur Tchebychev à M. Fuss sur un nouveau théorème relative aux nombres premiers continus dans les formes $4 n+1$ et $4 n+3$, Bull. Classe Phys. Acad. Imp. Sci. St. Petersburg 11 (1853), 208.

[5] H. Davenport, Multiplicative Number Theory, 2nd ed., Springer, 1980.

[6] H. L. Montgomery, The pair correlation of the zeros of the zeta function, in: Proc. Sympos. Pure Math. 24, Amer. Math. Soc., Providence, RI, 1973, 181-193.

[7] H. L. Montgomery and P. J. Weinberger, Notes on small class numbers, Acta Arith. 24 (1974), 529-542.

[8] A. E. Özlük, On the q-analogue of the pair correlation conjecture, J. Number Theory 59 (1996), 319-351.

[9] A. E. Özlük and C. Snyder, Small zeros of quadratic L-functions, Bull. Austral. Math. Soc. 47 (1993), 307-319. 
[10] P. Turán, On a New Method of Analysis and its Applications, Wiley, 1984.

[11] P. J. Weinberger, On small zeros of Dirichlet L-functions, Math. Comp. 29 (1975), 319-328.

Department of Mathematics and Statistics

University of Maine

Orono, ME 04469, U.S.A.

and

Research Institute of Mathematics, Orono

E-mail: ozluk@gauss.umemat.maine.edu snyder@gauss.umemat.maine.edu 